Brit. Heart f., 1966, 28, 557.

\title{
Comparison of Cardiorespiratory Responses to Graded Upright Exercise of Normal Men Aged 20-29 and 30-39*
}

\author{
JOHN S. HANSON + , BURTON S. TABAKIN, AND ARTHUR M. LEVY \\ From the Cardiopulmonary Laboratory, Department of Medicine, University of Vermont, \\ College of Medicine, Mary Fletcher Hospital, Burlington, Vermont, U.S.A.
}

Earlier communications from this laboratory have presented the cardiovascular and respiratory responses to graded treadmill exercise in normal men aged 20-29 years (Tabakin et al., 1964), observations during prolonged "steady state" exercise (Levy, Tabakin, and Hanson, 1961), an analysis of the effects of training in a group of distance runners (Tabakin, Hanson, and Levy, 1965), and a comparison of the responses in normal, sedentary men and athletes (Hanson and Tabakin, 1965). Simultaneous bilateral and "tandem" determinations (Hanson and Tabakin, 1964) have established the limits of reliability of the dyedilution method employed in these investigations.

The present report provides an extension of the above studies through comparison of the exercise reactions of 25 normal men aged 20-29 years and 25 normal men aged $30-39$ years. It is the purpose of this and subsequent communications to help fill a long-recognized and disconcerting void in the published reports concerning the cardiovascular behaviour of normal men during upright exercise of varying severity and the alterations in this performance with advancing age. Other reports will be concerned with the exercise profiles of the fifth and sixth decades of life, the 100 normal studies eventually forming a sound basis for comparison of individual exercise tests, as well as study and delineation of the functional defects associated with cardiac decompensation.

Received November 1, 1965.

* Supported by USPHS Research Grant HE-06121-03 and -04 of the National Heart Institute, Bethesda, Maryland, U.S.A.

t Recipient of USPHS Research Career Award (K-1-HE7248) of the National Heart Institute, Bethesda, Maryland, U.S.A.

\section{SubJECtS AND Methods}

Group I (20-29 years) and Group II (30-39 years) each comprised 25 male volunteers accepted for participation in the project in the order of their application without regard to previous or present exercise habits, body size, status of physical "conditioning", dietary, or smoking habits. Although it is true that the younger subjects, strictly from the standpoint of age, were as a whole more physically active than Group II, they were in no sense "athletes". An evaluation of each subject's exercise habits, as well as analysis of heart rates, oxygen utilization, etc., and comparison of these factors with those obtained in a trained group (Hanson and Tabakin, 1965), do not allow the conclusion that either Group I or Group II represented a non-random subject selection in this regard. It appears highly unlikely, therefore, that any of the significant intergroup differences in exercise response reported here may be related to a high degree of physical conditioning in one group. Group I consisted primarily of undergraduate and medical students, graduate students, and hospital workers, while Group II was composed largely of faculty members of the University of Vermont, graduate students, and hospital workers. For both groups a monetary incentive was provided, but its payment was not dependent on successful completion of any prescribed amount of work. Indeed, 3 subjects in Group II were not allowed to complete the most strenuous work load because of what were considered to be inordinate increases in heart rate or blood pressure in normal subjects for these particular degrees of exertion. Studies were interrupted when the heart rate reached 200 a minute, and systolic blood pressure reached 200-220 $\mathrm{mm}$. Hg. Medical histories and physical examinations were obtained in all subjects, and candidates were excluded who presented any previous history or current finding that might conceivably affect exercise results.

Detailed methodology has been presented in earlier reports (Hanson and Tabakin, 1964; Tabakin et al., 557 
technique using both the Waters 250-A and 300 densitometers and Cardiogreen. Intra-arterial pressure was measured with a Statham P23Db transducer and amplification of this signal in the Electronics-for-Medicine DR-8 recorder. Minute volume of ventilation, oxygen utilization, carbon dioxide elimination, and oxygen ventilatory equivalent were measured and calculated by standard methods from expired gas collections. Peripheral vascular resistance was estimated from cardiac output and mean arterial pressure. Left ventricular work index and stroke work index were calculated as the products of cardiac and stroke indices respectively, mean arterial pressure and the constant 13.6/1000.

Measurement and calculation of these parameters were made with the subject recumbent at rest, standing at rest, walking at 3 miles per hour on the level treadmill and at treadmill elevations of $4^{\circ}, 8^{\circ}, 12^{\circ}, 14^{\circ}$. Determinations and collections were made after 4 minutes of exercise for the level, $4^{\circ}$, and $8^{\circ}$ walks, and after 2-3 minutes for the $12^{\circ}$ and $14^{\circ}$ walks. Between each exercise period subjects were allowed to rest sitting until their heart rates approximated the original resting recumbent values, thus avoiding fallacious variations in results secondary to prolonged work periods (Levy et al., 1961).

Appropriate statistical analysis of results was accomplished with the IBM 1620 Data Processor employing standard programmes. For the purposes of this study adherence to the following $p$-value interpretations were made: $<0.05=$ probably significant; $<0.01=$ significant; $<0.001=$ highly significant. On the basis of all the data collected during standing resting and exercise determinations, comprising well over 5,000 individual values, simple linear and multiple variable regression equations and coefficients of correlation have been established for the most important variables. Tables III, IV, and V present this material which may be utilized as standards of exercise response in normal men between the ages of 20 and 39, as well as predictors for any one parameter from other perhaps simpler measurements. The multiple variable regression and correlation data were obtained with the two-stage, stepwise IBM programme number $6 \cdot 0 \cdot 057$, compiled by $E$. Vernon Griffith.

TABLE I

PHYSICAL CHARACTERISTICS OF GROUPS I AND II

\begin{tabular}{c|c|c|c|c}
\hline & $\begin{array}{c}\text { Age } \\
(\mathrm{yr} .)\end{array}$ & $\begin{array}{c}\text { Height } \\
(\mathrm{cm} .)\end{array}$ & $\begin{array}{c}\text { Weight } \\
(\mathrm{kg} .)\end{array}$ & $\begin{array}{c}\text { BSA } \\
\left(\mathrm{m} .{ }^{2}\right)\end{array}$ \\
\hline $\begin{array}{c}\text { Group I } \\
\mathrm{n}=25\end{array}\left\{\begin{array}{l}\text { mean } \\
\text { range }\end{array}\right.$ & $\begin{array}{c}23 \\
(20-29)\end{array}$ & $\begin{array}{c}176 \\
(145-188)\end{array}$ & $\begin{array}{c}77 \\
(58-94)\end{array}$ & $\begin{array}{c}1 \cdot 92 \\
(1 \cdot 59-2 \cdot 12)\end{array}$ \\
$\begin{array}{c}\text { Group II } \\
\mathbf{n}=25\end{array}\left\{\begin{array}{l}\text { mean } \\
\text { range }\end{array}\right.$ & $\begin{array}{c}33 \\
(30-39)\end{array}$ & $\begin{array}{c}176 \\
(159-200)\end{array}$ & $\begin{array}{c}80 \\
(66-97)\end{array}$ & $\begin{array}{c}1.96 \\
(1 \cdot 70-2 \cdot 28)\end{array}$ \\
\hline
\end{tabular}

\section{Results}

It is important to note that the mean anthropological data for the 2 groups is almost identical (Table I). The difference in mean body surface is only $0.04 \mathrm{~m}^{2}$., and therefore the physiological data presented here are readily comparable either in their raw form or as indices. Likewise, the mean work loads for both groups are insignificantly different with a maximum variance of only 4.5 per cent at the $14^{\circ}$ treadmill walk. Disparities in mean group exercise responses must, therefore, represent true differences in adaptation to exercise.

Major age dissimilarities in the magnitude of cardiac output attained for each exercise load were not observed in the present material. Group II had a consistently lower mean value for this variable with the exception of the $4^{\circ}$ and $14^{\circ}$ walks, where group values were nearly identical. The greatest difference, amounting to $2.05 \mathrm{l}$./min., appeared at the $12^{\circ}$ treadmill inclination, but its significance could not be established statistically. For the remaining determination intergroup variation was in the relatively narrow range of $270-670 \mathrm{ml} . / \mathrm{min}$. These results, as well as those following, are presented in tabular form in Table II. This similarity is also represented graphically in Fig. 1 with cardiac output plotted as a function of oxygen utilization.

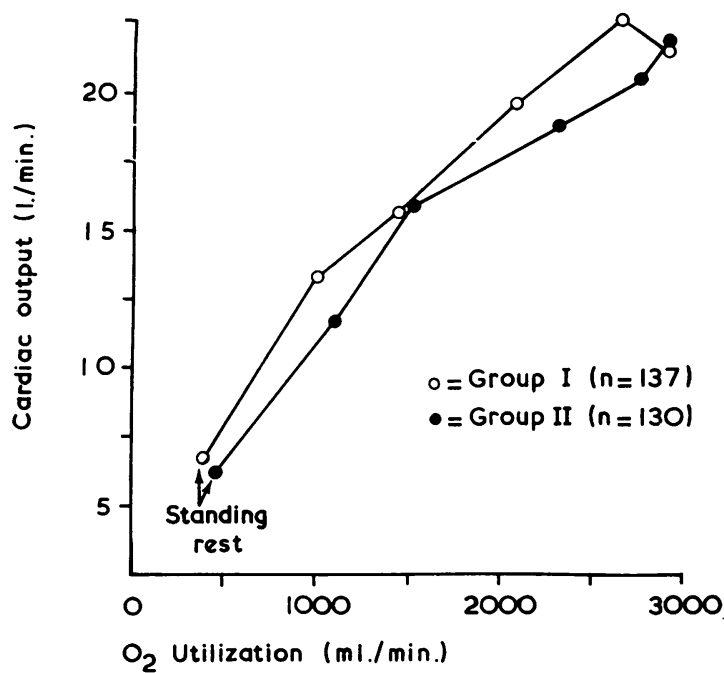

Fig. 1.-Relation of mean values for cardiac output to those for oxygen utilization for subjects standing at rest and at 5 levels of treadmill exercise.

It is readily apparent that for any given level of oxygen uptake Group II exhibits a moderately lower output, this divergence becoming more marked at the higher work loads, only to be abolished at peak exercise.

Tables III and IV present, respectively, the linear and multiple regression equations for prediction of cardiac output from heart rate, minute volume of ventilation, oxygen utilization, and carbon dioxide elimination. It may be seen that for both Groups I 
TABLE II

COMPARISON OF MEAN VALUES FOR ALL VARIABLES MEASURED AT REST AND DURING EXERCISE

\begin{tabular}{|c|c|c|c|c|c|c|c|c|c|c|c|c|c|c|c|c|}
\hline \multirow[t]{2}{*}{$\begin{array}{c}\text { Exercise } \\
\text { level }\end{array}$} & \multirow[t]{2}{*}{ Group } & \multirow{2}{*}{$\begin{array}{l}\text { Heart } \\
\text { rate } \\
\text { (beats/ } \\
\text { (min.) }\end{array}$} & \multirow{2}{*}{$\begin{array}{c}\text { Car- } \\
\text { diac } \\
\text { out- } \\
\text { put } \\
(1 . / \\
\text { min.) }\end{array}$} & \multirow{2}{*}{$\begin{array}{c}\text { Car- } \\
\text { diac } \\
\text { index } \\
(1 . / \\
\text { min./ } \\
\left.\mathrm{m.}^{2}\right)\end{array}$} & \multirow{2}{*}{$\begin{array}{c}\text { Stroke } \\
\text { vol- } \\
\text { ume } \\
(\mathrm{ml} .)\end{array}$} & \multirow{2}{*}{$\begin{array}{c}\text { Min- } \\
\text { ute } \\
\text { vol- } \\
\text { ume(1./ } \\
\text { min.) } \\
\text { BTPS }\end{array}$} & \multirow{2}{*}{$\begin{array}{c}\mathrm{O}_{2} \\
\text { Utili- } \\
\text { zation } \\
\text { (ml./ } \\
\text { min.) } \\
\text { STPD }\end{array}$} & \multirow{2}{*}{$\begin{array}{c}\mathrm{CO}_{2} \\
\text { elimi- } \\
\text { nation } \\
\text { (ml./ } \\
\text { min.) } \\
\text { STPD }\end{array}$} & \multirow{2}{*}{$\begin{array}{c}\text { Work } \\
\text { load } \\
\text { (kg.m./ } \\
\text { min.) }\end{array}$} & \multicolumn{3}{|c|}{$\underset{(\mathrm{mm} . \mathrm{Hg})}{\text { Blood Pressure }}$} & \multirow{2}{*}{$\begin{array}{c}\text { PVR } \\
\text { dynes } \\
\text { (cm. } \\
\left.\text { sec. }^{-5}\right)\end{array}$} & \multirow[t]{2}{*}{$\begin{array}{c}\mathrm{O}_{2} \mathrm{~V} \\
1 . / 1 \mathrm{O}_{2}\end{array}$} & \multirow{2}{*}{$\underset{\substack{\text { LVWI } \\
\text { m.m. } \\
\text { m.2) }}}{\mid}$} & \multirow{2}{*}{$\mid \begin{array}{c}\text { LVSWI } \\
\text { (mg./ } \\
\left.\mathrm{m.}^{2}\right)\end{array}$} \\
\hline & & & & & & & & & & Syst. & Diast. & Mean & & & & \\
\hline $\begin{array}{l}\text { Resting } \\
\text { recumbent }\end{array}$ & 1 & $\begin{array}{l}72 \\
82\end{array}$ & $\begin{array}{l}8 \cdot 03 \\
7 \cdot 76\end{array}$ & $\begin{array}{l}4 \cdot 20 \\
3 \cdot 96\end{array}$ & $\begin{array}{l}112 \\
112\end{array}$ & $\begin{array}{l}9 \cdot 60 \\
9 \cdot 88\end{array}$ & $\begin{array}{l}300 \\
300\end{array}$ & $\begin{array}{l}250 \\
257\end{array}$ & - & $\begin{array}{l}124 \\
132 \\
\end{array}$ & $\begin{array}{l}67 \\
72 \\
\end{array}$ & $\begin{array}{l}82 \\
90 \\
\end{array}$ & $\begin{array}{r}849 \\
956 \\
\end{array}$ & $\begin{array}{r}25 \\
27 \\
\end{array}$ & $\begin{array}{r}4 \cdot 69 \\
4 \cdot 88 \\
\end{array}$ & $\begin{array}{l}64 \cdot 68 \\
70 \cdot 18 \\
\end{array}$ \\
\hline $\begin{array}{l}\text { Resting } \\
\text { Standing }\end{array}$ & II & $\begin{array}{l}109 \\
108\end{array}$ & $\begin{array}{l}6 \cdot 73 \\
6 \cdot 18\end{array}$ & $\begin{array}{l}3 \cdot 50 \\
3 \cdot 14\end{array}$ & $\begin{array}{l}63 \\
60\end{array}$ & $\begin{array}{l}12 \cdot 21 \\
14 \cdot 09 \\
\end{array}$ & $\begin{array}{l}423 \\
465\end{array}$ & $\begin{array}{l}285 \\
353\end{array}$ & - & $\begin{array}{l}109 \\
109\end{array}$ & $\begin{array}{l}65 \\
66\end{array}$ & $\begin{array}{l}80 \\
81\end{array}$ & $\begin{array}{l}1013 \\
1132\end{array}$ & $\begin{array}{l}24 \\
25\end{array}$ & $\begin{array}{l}3 \cdot 81 \\
3 \cdot 47\end{array}$ & $\begin{array}{l}35 \cdot 90 \\
33.57\end{array}$ \\
\hline $\begin{array}{l}\text { Level } \\
\text { treadmill walk }\end{array}$ & II & $\begin{array}{l}107 \\
101\end{array}$ & $\begin{array}{l}13 \cdot 31 \\
12.65 \\
\end{array}$ & $\begin{array}{l}6.93 \\
6.45\end{array}$ & $\begin{array}{l}125 \\
125\end{array}$ & $\begin{array}{l}24 \cdot 23 \\
24 \cdot 93 \\
\end{array}$ & $\begin{array}{l}1040 \\
1119\end{array}$ & $\begin{array}{l}773 \\
814 \\
\end{array}$ & - & $\begin{array}{l}139 \\
152\end{array}$ & $\begin{array}{l}60 \\
71 \\
\end{array}$ & $\begin{array}{l}85 \\
95 \\
\end{array}$ & $\begin{array}{r}536 \\
611 \\
\end{array}$ & $\begin{array}{l}19 \\
18 \\
\end{array}$ & $\begin{array}{l}8 \cdot 01 \\
8 \cdot 40 \\
\end{array}$ & $\begin{array}{l}75 \cdot 14 \\
82 \cdot 25 \\
\end{array}$ \\
\hline $4^{\circ}$ & II & $\begin{array}{l}123 \\
119\end{array}$ & $\begin{array}{l}15.51 \\
15.85 \\
\end{array}$ & $\begin{array}{l}8 \cdot 12 \\
8 \cdot 10\end{array}$ & $\begin{array}{l}127 \\
134\end{array}$ & $\begin{array}{l}32 \cdot 08 \\
34 \cdot 24\end{array}$ & $\begin{array}{l}1480 \\
1600\end{array}$ & $\begin{array}{l}1172 \\
1232\end{array}$ & $\begin{array}{l}428 \\
448\end{array}$ & $\begin{array}{l}140 \\
153\end{array}$ & $\begin{array}{l}57 \\
69 \\
\end{array}$ & $\begin{array}{l}83 \\
95\end{array}$ & $\begin{array}{l}442 \\
494\end{array}$ & $\begin{array}{l}18 \\
18\end{array}$ & $\begin{array}{r}9 \cdot 17 \\
10 \cdot 45\end{array}$ & $\begin{array}{l}74.50 \\
88.03\end{array}$ \\
\hline $8^{\circ}$ & I & $\begin{array}{l}152 \\
148\end{array}$ & $\begin{array}{l}19.47 \\
18.80\end{array}$ & $\begin{array}{r}10 \cdot 14 \\
9.62 \\
\end{array}$ & $\begin{array}{l}133 \\
128\end{array}$ & \begin{tabular}{|l|}
$45 \cdot 87$ \\
$50 \cdot 88$ \\
\end{tabular} & $\begin{array}{l}2117 \\
2341\end{array}$ & $\begin{array}{l}1710 \\
1903\end{array}$ & $\begin{array}{l}856 \\
895\end{array}$ & $\begin{array}{l}134 \\
166\end{array}$ & $\begin{array}{l}55 \\
73 \\
\end{array}$ & $\begin{array}{r}78 \\
102 \\
\end{array}$ & $\begin{array}{r}335 \\
442 \\
\end{array}$ & $\begin{array}{r}17 \\
18 \\
\end{array}$ & $\begin{array}{l}10 \cdot 76 \\
13 \cdot 40 \\
\end{array}$ & $\begin{array}{l}73.20 \\
91.08 \\
\end{array}$ \\
\hline $12^{\circ}$ & II & $\begin{array}{l}168 \\
170\end{array}$ & $\begin{array}{l}22.57 \\
20.52 \\
\end{array}$ & $\begin{array}{l}11 \cdot 71 \\
10 \cdot 51\end{array}$ & $\begin{array}{l}136 \\
121 \\
\end{array}$ & $\begin{array}{l}59 \cdot 10 \\
67 \cdot 01 \\
\end{array}$ & $\begin{array}{l}2676 \\
2779\end{array}$ & $\begin{array}{l}2228 \\
2406\end{array}$ & $\begin{array}{l}1290 \\
1337\end{array}$ & $\begin{array}{l}137 \\
174 \\
\end{array}$ & $\begin{array}{l}53 \\
75 \\
\end{array}$ & $\begin{array}{r}77 \\
107 \\
\end{array}$ & $\begin{array}{l}283 \\
435 \\
\end{array}$ & $\begin{array}{r}18 \\
20 \\
\end{array}$ & $\begin{array}{l}12 \cdot 26 \\
15 \cdot 23 \\
\end{array}$ & $\begin{array}{l}74 \cdot 35 \\
90 \cdot 15\end{array}$ \\
\hline $14^{\circ}$ & I & $\begin{array}{l}176 \\
175\end{array}$ & $\begin{array}{l}21 \cdot 55 \\
21.87\end{array}$ & $\begin{array}{l}11.09 \\
11.13\end{array}$ & $\begin{array}{l}122 \\
127\end{array}$ & $\begin{array}{l}68 \cdot 04 \\
71 \cdot 35\end{array}$ & $\begin{array}{l}2948 \\
2949\end{array}$ & $\begin{array}{l}2406 \\
2517\end{array}$ & $\begin{array}{l}1501 \\
1570\end{array}$ & $\begin{array}{l}132 \\
168\end{array}$ & $\begin{array}{l}49 \\
76\end{array}$ & $\begin{array}{r}75 \\
106\end{array}$ & $\begin{array}{l}286 \\
416\end{array}$ & $\begin{array}{l}19 \\
20\end{array}$ & $\begin{array}{l}11 \cdot 31 \\
16.06\end{array}$ & $\begin{array}{l}65 \cdot 29 \\
93 \cdot 33\end{array}$ \\
\hline
\end{tabular}

LVWI = left ventricular work index.

LVSWI = left ventricular stroke work index.

and II the correlation coefficient of cardiac output is only slightly improved by multiple variable analysis as compared to simple linear correlation. For example, the correlation coefficient for cardiac output with oxygen utilization of 0.77 in Group I

\section{TABLE III}

SIMPLE LINEAR REGRESSION EQUATIONS AND CORRELATION COEFFICIENTS CALCULATED FROM STANDING, RESTING, AND EXERCISING MEASUREMENTS

\begin{tabular}{|c|c|c|c|}
\hline$y$ & $x$ & Regression equation & $r$ \\
\hline $\begin{array}{l}\text { Cardiac putput } \\
\text { Cardiac output } \\
\text { Cardiac output } \\
\text { Cardiac output } \\
\text { Cardiac output } \\
\\
\text { Heart rate } \\
\text { Minute volume } \\
\mathrm{O}_{2} \text { uptake } \\
\mathrm{CO}_{2} \text { elimination } \\
\mathrm{Mlinute} \text { volume } \\
\mathrm{O}_{2} \text { uptake } \\
\mathrm{CO}_{2} \text { elimination } \\
\text { Peripheral } \\
\text { resistance }\end{array}$ & $\begin{array}{l}\text { Age 2 } \\
\text { Heart rate } \\
\text { Work load } \\
\text { Minute volume } \\
\mathrm{O}_{2} \text { uptake } \\
\mathrm{CO}_{2} \\
\text { elimination } \\
\text { Work load } \\
\text { Work load } \\
\text { Work load } \\
\text { Work load } \\
\text { Heart rate } \\
\text { Heart rate } \\
\text { Heart rate } \\
\mathrm{O}_{2} \text { uptake }\end{array}$ & $\begin{array}{l}-29 \text { years } \\
y=-1 \cdot 48+0 \cdot 13 x \\
y=-0 \cdot 22+0 \cdot 020 x \\
y=-0 \cdot 37+0 \cdot 422 x \\
y=0 \cdot 42+0 \cdot 0091 x \\
y=2 \cdot 81+0 \cdot 0097 x \\
y=70 \cdot 8+0 \cdot 083 x \\
y=1 \cdot 3+0 \cdot 0495 x \\
y=670 \cdot 8+1 \cdot 61 x \\
y=105 \cdot 8+1 \cdot 75 x \\
y=-34 \cdot 0+0 \cdot 54 x \\
y=-1343+22 \cdot 5 x \\
y=-1533+21 \cdot 4 x \\
y=1248-0 \cdot 435 x\end{array}$ & $\begin{array}{l}0.68 \\
0.56 \\
0.72 \\
0.77 \\
\\
0.80 \\
0 \cdot 76 \\
0.80 \\
0.92 \\
0.82 \\
0.83 \\
0.81 \\
0.85 \\
-0.72\end{array}$ \\
\hline $\begin{array}{l}\text { Cardiac ouput } \\
\text { Cardiac output } \\
\text { Cardiac output } \\
\text { Cardiac output } \\
\text { Cardiac output } \\
\text { Heart rate } \\
\text { Minute volume } \\
\mathrm{O}_{2} \text { uptake } \\
\mathrm{CO}_{2} \text { elimination } \\
\mathrm{Minute} \text { volume } \\
\mathrm{O}_{2} \text { uptake } \\
\mathrm{CO}_{2} \text { elimination } \\
\text { Peripheral } \\
\text { resistance }\end{array}$ & $\begin{array}{l}\text { Heart rate } \\
\text { Work load } \\
\text { Work } \\
\text { Minute volume } \\
\mathrm{O}_{2} \text { uptake } \\
\mathrm{CO}_{2} \\
\text { elimination } \\
\text { Work load } \\
\text { Work load } \\
\text { Work load } \\
\text { Work load } \\
\text { Heart rate } \\
\text { Heart rate } \\
\text { Heart rate } \\
\mathrm{O}_{2} \text { uptake }\end{array}$ & $\begin{array}{l}-39 \text { years } \\
y=0.28+0.12 x \\
y=0.04+0.019 x \\
y=0 \cdot 21+0.364 x \\
y=-0 \cdot 20+0 \cdot 0086 x \\
y=0 \cdot 78+0 \cdot 0099 x \\
y=76 \cdot 1+0.074 x \\
y=3 \cdot 54+0 \cdot 0498 x \\
y=732+1 \cdot 59 x \\
y=-34 \cdot 3+0 \cdot 58 x \\
y=-34 \cdot 3+0 \cdot 58 x \\
y=-1256+23 \cdot 2 x \\
y=-1378+21 \cdot 5 x \\
y=1483-0.476 x\end{array}$ & $\begin{array}{l}0.67 \\
0.53 \\
0 \cdot 71 \\
0 \cdot 76 \\
0.73 \\
0 \cdot 81 \\
0.85 \\
0.89 \\
0 \cdot 82 \\
0 \cdot 82 \\
0 \cdot 84 \\
0.87 \\
-0.62\end{array}$ \\
\hline
\end{tabular}

closely approximates that of cardiac output with 4 independent variables $(0 \cdot 82)$. However, the reliability of cardiac output prediction is of course improved by utilization of 3 or 4 independent variables, as evidenced by lower residual standard deviations for these equations.

Resting recumbent pulse rate for Group I was 10 beats/min. lower than Group II, but this is the maximum variation seen in the entire study. For the other 6 measurements differences ranged from only $1-6$ beats/min. with almost identical heart rates for both groups during all exercise.

In view of the preceding, it is not surprising that a strong similarity in stroke volume response was also observed. As seen in Fig. 2 the initiation of exercise is attended by a 100 per cent increase of the stroke volume maintained standing at rest, and thereafter increase in work load has little effect on the magnitude of this variable. The greatest group difference in stroke volume values is seen at the $12^{\circ}$ level, but even this was statistically insignificant. Since the pulse responses were almost identical for this work load, this difference exists solely on the basis of the previously mentioned inequality in mean cardiac output.

A striking and highly significant delineation of the two groups is obtained through consideration of the blood pressure and peripheral vascular resistance responses to exercise. Ventricular work and stroke work indices, dependent in part on mean arterial pressure, also provide this clear-cut group distinction at the 4 highest exercise levels.

With the sole exception of the values obtained at 
TABLE IV

CARDIAC OUPUT $(y)$ PREDICTED FOR GROUPS I AND II FROM HEART RATE (HR, beats/min.), MINUTE VOLUME OF VENTILATION (MV, 1./min.), OXYGEN UTILIZATION (O, ml./min.), CARBON DIOXIDE ELIMINATION (CO 2 ml./min.)

\begin{tabular}{|c|c|c|c|}
\hline $\begin{array}{l}\text { Independent } \\
\text { variable }\end{array}$ & Group & Regression equation & $\begin{array}{c}\text { Multiple } \\
\text { correlation } \\
\text { coefficient }\end{array}$ \\
\hline $\begin{array}{l}\operatorname{HR}\left(x_{1}\right) \\
\operatorname{MV}\left(x_{2}\right)\end{array}$ & II & $\begin{array}{l}y=3.61+0.051 x_{1}+0.15 x_{2} \\
y=4.69+0.044 x_{1}+0.13 x_{2}\end{array}$ & $\begin{array}{l}0.73 \\
0 \cdot 72\end{array}$ \\
\hline $\begin{array}{l}\mathrm{HR}\left(x_{1}\right) \\
\mathrm{O}_{2}\left(x_{2}\right)\end{array}$ & II & $\begin{array}{l}y=4.59+0.029 x_{1}+0.005 x_{2} \\
y=6.01+0.012 x_{1}+0.005 x_{2}\end{array}$ & $\begin{array}{l}0.77 \\
0.76\end{array}$ \\
\hline$\underset{\mathrm{CO}_{2}\left(x_{2}\right)}{\mathrm{HR}\left(x_{1}\right)}$ & I & $\begin{array}{l}y=8 \cdot 28-0.006 x_{1}+0.006 x_{2} \\
y=6.54+0.02 x_{1}+0.005 x_{2}\end{array}$ & $\begin{array}{l}0.80 \\
0.73\end{array}$ \\
\hline $\begin{array}{l}\operatorname{MV}\left(x_{1}\right) \\
\mathrm{O}_{2}\left(x_{2}\right)\end{array}$ & $\begin{array}{l}\text { I } \\
\text { II }\end{array}$ & $\begin{array}{l}y=7 \cdot 11-0.041 x_{1}+0.006 x_{2} \\
y=6.91-0.003 x_{1}+0.005 x_{2}\end{array}$ & $\begin{array}{l}0.77 \\
0.76\end{array}$ \\
\hline $\begin{array}{l}\mathrm{MV}\left(x_{1}\right) \\
\mathrm{CO}_{2}\left(x_{2}\right)\end{array}$ & II & $\begin{array}{l}y=8 \cdot 17-0.065 x_{1}+0.008 x_{2} \\
y=8.07+0.038 x_{1}+0.004 x_{2}\end{array}$ & $\begin{array}{l}0.81 \\
0 \cdot 73\end{array}$ \\
\hline $\begin{array}{l}\mathrm{O}_{2}\left(x_{1}\right) \\
\mathrm{CO}_{2}\left(x_{2}\right)\end{array}$ & II & $\begin{array}{l}y=7.46+0.001 x_{1}+0.005 x_{2} \\
y=6.77+0.006 x_{1}-0.001 x_{2}\end{array}$ & $\begin{array}{l}0.80 \\
0.76 \\
\end{array}$ \\
\hline $\begin{array}{l}\operatorname{HR}\left(x_{1}\right) \\
\operatorname{MV}\left(x_{2}\right) \\
\mathrm{O}_{2}\left(x_{3}\right)\end{array}$ & II & $\begin{array}{l}y=3.87+0.038 x_{1}-0.077 x_{2}+0.006 x_{3} \\
y=5.94+0.013 x_{1}-0.008 x_{2}+0.005 x_{3}\end{array}$ & $\begin{array}{l}0.78 \\
0.76\end{array}$ \\
\hline $\begin{array}{l}\operatorname{HR}\left(x_{1}\right) \\
\operatorname{MV}\left(x_{2}\right) \\
\mathrm{CO}_{2}\left(x_{3}\right)\end{array}$ & II & $\begin{array}{l}y=8 \cdot 10+0.0008 x_{1}-0.067 x_{2}+0.008 x_{3} \\
y=6.42+0.02 x_{1}+0.039 x_{2}+0.004 x_{3}\end{array}$ & $\begin{array}{l}0.81 \\
0.73\end{array}$ \\
\hline $\begin{array}{l}\mathrm{HR}\left(x_{1}\right) \\
\mathrm{O}_{2}\left(x_{2}\right) \\
\mathrm{CO}_{2}\left(x_{3}\right)\end{array}$ & II & $\begin{array}{l}y=8 \cdot 10-0.007 x_{1}+0.001 x_{2}+0.005 x_{3} \\
y=5 \cdot 16+0.02 x_{1}+0.006 x_{2}-0.002 x_{3}\end{array}$ & $\begin{array}{l}0.80 \\
0 \cdot 76\end{array}$ \\
\hline $\begin{array}{l}\operatorname{MV}\left(x_{1}\right) \\
\mathrm{O}_{3}\left(x_{2}\right) \\
\mathrm{CO}_{2}\left(x_{3}\right)\end{array}$ & II & $\begin{array}{l}y=7.71-0.14 x_{1}+0.003 x_{2}+0.006 x_{3} \\
y=6.74+0.015 x_{1}+0.006 x_{2}-0.002 x_{3}\end{array}$ & $\begin{array}{l}0.82 \\
0.76\end{array}$ \\
\hline $\begin{array}{l}\operatorname{HR}\left(x_{1}\right) \\
M V\left(x_{2}\right) \\
\mathrm{O}_{2}\left(x_{3}\right) \\
\mathrm{CO}_{2}\left(x_{4}\right)\end{array}$ & $\begin{array}{l}\text { I } \\
\text { II }\end{array}$ & $\begin{array}{l}y=7.37+0.004 x_{1}-0.14 x_{2}+0.003 x_{3}+0.006 x_{4} \\
y=5.13+0.02 x_{1}+0.016 x_{2}+0.006 x_{3}-0.002 x_{4}\end{array}$ & $\begin{array}{l}0.82 \\
0.76\end{array}$ \\
\hline
\end{tabular}

rest, group differences in systolic, diastolic, and mean intra-arterial pressures become progressively larger with each increase in exercise load. Stat-

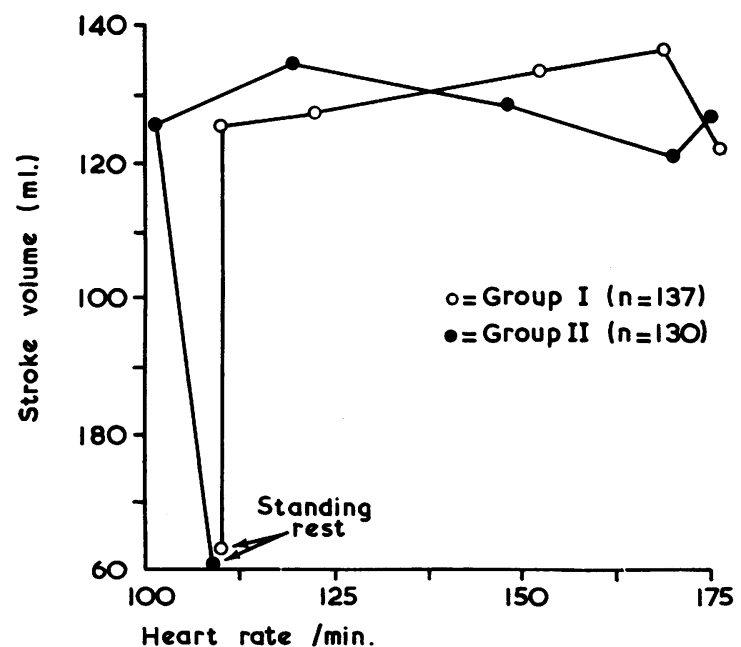

Fig. 2.-Mean stroke volume in relation to mean heart rate with subjects standing at rest and during progressively severe exercise. istical significance of these differences also increases in step-wise fashion for each increase in treadmill elevation. This marked intergroup variation may be appreciated from Fig. 3. Whereas diastolic and mean pressures in Group I decrease with each successive exercise load, Group II is seen to maintain an almost constant diastolic pressure but a steadily rising mean pressure. This latter observation is related to the constantly increasing exercise systolic pressure of Group II as compared with the virtual systolic plateau seen in Group I.

Related to these considerations, an interesting relationship of blood pressure with ventilatory variables becomes apparent in analysis of the statistical data. Although simple correlations of systolic, diastolic, and mean arterial pressure with minute volume, oxygen utilization, and carbon dioxide elimination were of a relatively low order in Group I $(r=0 \cdot 18-0.44)$, more significant values were obtained in Group II for correlation of mean pressure $(r=0.60-0.64)$ and systolic pressure $(r=0.55-0.65)$ with these respiratory parameters. A similar phenomenon was also apparent in the multiple variable correlations of mean pressure with heart rate and ventilatory data. Whereas 
TABLE V

GROUP II MEAN INTRA-ARTERIAL BLOOD PRESSURE ( $y$ ) IN RELATION TO HEART RATE (HR, beats/min.), MINUTE VOLUME OF VENTILATION (MV, $1.1 \mathrm{~min}$ ), OXYGEN UTILIZATION $\left(\mathrm{O}_{2}, \mathrm{ml} . / \mathrm{min}\right.$.), CARBON DIOXIDE ELIMINATION $\left(\mathrm{CO}_{2}, \mathrm{ml}\right.$./min.).

\begin{tabular}{|c|c|c|c|}
\hline $\begin{array}{l}\text { Independent } \\
\text { variable }\end{array}$ & Regression equation & $\begin{array}{l}\text { Correlation } \\
\text { coefficient }\end{array}$ & $\begin{array}{l}\text { Multiple } \\
\text { correlation } \\
\text { coefficient }\end{array}$ \\
\hline HR & $y=70 \cdot 7+0.204 x$ & 0.52 & \\
\hline MV & $y=84 \cdot 3+0 \cdot 324 x$ & 0.58 & \\
\hline $\mathrm{O}_{2}$ & $y=82 \cdot 9+0 \cdot 008 x$ & 0.59 & \\
\hline $\mathrm{CO}_{2}$ & $y=83.5+0.0097 x$ & 0.62 & \\
\hline $\begin{array}{l}\operatorname{HR}\left(x_{1}\right) \\
\operatorname{MV}\left(x_{2}\right)\end{array}$ & $y=80.0+0.05 x_{1}+0.266 x_{2}$ & & 0.59 \\
\hline $\begin{array}{l}\mathrm{HR}\left(x_{1}\right) \\
\mathrm{O}_{2}\left(x_{2}\right) \\
\mathrm{HR}^{2}\left(x_{1}\right) \\
\mathrm{CO}_{2}\left(x_{2}\right)\end{array}$ & $\begin{array}{l}y=79.9+0.037 x_{1}+0.007 x_{2} \\
y=85.6-0.025 x_{1}+0.011 x_{2}\end{array}$ & & $\begin{array}{l}0.59 \\
0.62\end{array}$ \\
\hline $\begin{array}{l}\operatorname{MV}\left(x_{1}\right) \\
\mathrm{O}_{2}\left(x_{2}\right)\end{array}$ & $y=83 \cdot 0+0 \cdot 16 x_{1}+0 \cdot 0045 x_{2}$ & & 0.60 \\
\hline $\begin{array}{l}\operatorname{MV}\left(x_{1}\right) \\
\mathrm{CO}_{2}\left(x_{2}\right)\end{array}$ & $y=83.6-0.045 x_{1}+0.011 x_{2}$ & & 062 \\
\hline $\begin{array}{l}\mathrm{O}_{2}\left(x_{1}\right) \\
\mathrm{CO}_{2}\left(x_{2}\right) \\
\end{array}$ & $y=84 \cdot 6-0.0048 x_{1}+0.015 x_{2}$ & & 0.63 \\
\hline $\begin{array}{l}\operatorname{HR}\left(x_{1}\right) \\
\operatorname{MV}\left(x_{2}\right) \\
\mathrm{O}_{2}\left(x_{3}\right)\end{array}$ & $y=81 \cdot 0+0.025 x_{1}+0.15 x_{2}+0.004 x_{3}$ & & 0.60 \\
\hline $\begin{array}{l}\operatorname{HR}\left(x_{1}\right) \\
\operatorname{MV}\left(x_{2}\right) \\
\mathrm{CO}_{2}\left(x_{3}\right)\end{array}$ & $y=85.7-0.025 x_{1}-0.046 x_{2}+0.012 x_{3}$ & & 0.62 \\
\hline $\begin{array}{l}\mathrm{HR}\left(x_{1}\right) \\
\mathrm{O}_{2}\left(x_{2}\right) \\
\mathrm{CO}_{2}\left(x_{3}\right)\end{array}$ & $y=86.7-0.026 x_{1}-0.005 x_{2}+0.016 x_{3}$ & & 0.63 \\
\hline $\begin{array}{l}\mathrm{MV}\left(x_{1}\right) \\
\mathrm{O}_{2}\left(x_{2}\right) \\
\mathrm{CO}_{2}\left(x_{3}\right) \\
\end{array}$ & $y=84.6-0.027 x_{1}-0.005 x_{2}+0.015 x_{3}$ & & 0.63 \\
\hline $\begin{array}{l}\operatorname{HR}\left(x_{1}\right) \\
\mathrm{MV}\left(x_{2}\right) \\
\mathrm{O}_{2}\left(x_{3}\right) \\
\mathrm{CO}_{2}\left(x_{4}\right)\end{array}$ & $y=86.8=0.026 x_{1}-0.028 x_{2}-0.005 x_{3}+0.016 x_{4}$ & & 0.63 \\
\hline
\end{tabular}

coefficients of all possible correlations were less than 0.50 for Group $I$, the values varied between 0.52-0.63 for Group II (Table V). Predictive multiple regression data for mean arterial pressure in the 30-39-year-old men are presented, since this variable may be applied to calculations of vascular resistance and ventricular work indices. As in the case of the corresponding equations for cardiac output, the predictive accuracy increases as more independent variables are employed. All correlation values are highly significant. Group comparisons with regard to peripheral vascular resistance are illustrated in Fig. 4 together with the indicators of statistical significance of the group differences. It should be noted that whereas Group I manifests an almost linear decline in resistance from the inception of exercise through the $12^{\circ}$ walk, Group II's mean values reach an asymptote at $8^{\circ}$.

Employing heart rate as an indicator of exercise

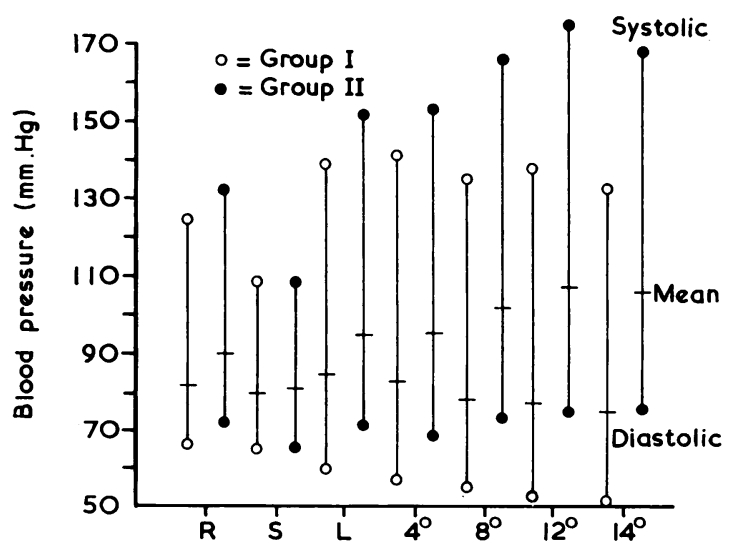

Fig. 3.-Group mean values for systolic, diastolic, and mean intra-arterial pressure. $R=$ resting recumbent; $S=$ standing resting; $L=$ level treadmill walk; $4^{\circ}, 8^{\circ}, 12^{\circ}, 14^{\circ}=$ respective treadmill elevations. 


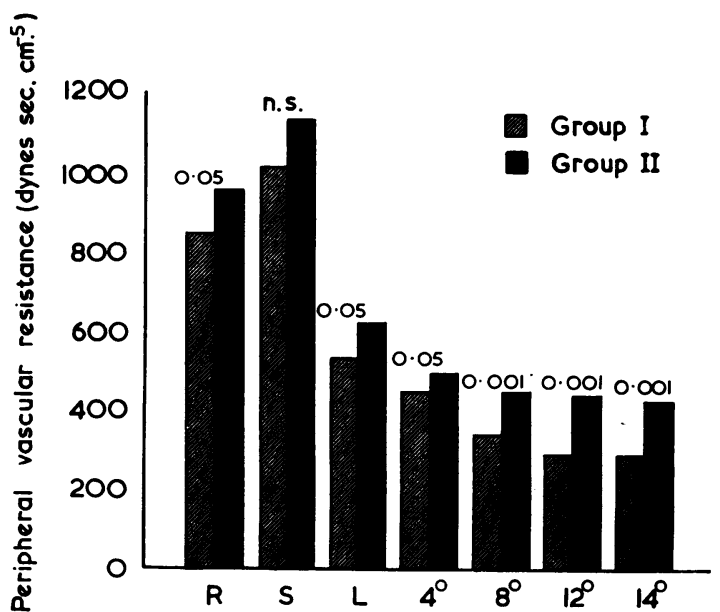

Fig. 4.-Mean vascular resistance at rest and during exercise. Column designations as in Fig. 3. p-values of statistical significance of group differences appear above each column pair.

severity, Fig. 5 and 6 illustrate the increased ventricular stress imposed on Group II at all grades of work as a result of comparatively raised arterial pressure. Statistical significance of the group differences in work and stroke work indices was established for the $8^{\circ}, 12^{\circ}$, and $14^{\circ}$ treadmill elevations. In addition, Group I's stroke work index was probably significantly lower than that of Group II at $4^{\circ}$.

Comparison of the ventilatory responses indicates that the older men evidenced consistently higher values for minute volume, oxygen utilization, and carbon dioxide elimination both at rest and for

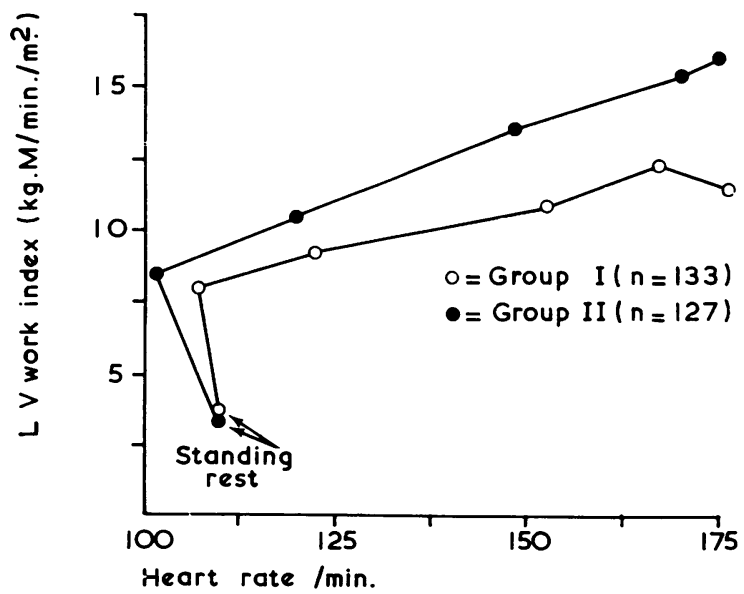

Fig. 5.-Plot of mean left ventricular work index versus heart rate for standing rest and at 5 levels of upright exercise. all exercise. In terms of volume of ventilation per litre of oxygen utilization, the oxygen ventilatory equivalent, Group II's performance was again marked by higher values than Group I for 5 of the 7 measurements. Despite these trends no statistical significance existed for the comparison of group differences.

\section{Discussion}

Numerous reviews of available data (Åstrand, 1956; Shock, 1964; Wade and Bishop, 1962) attest to the singular lack of systematically obtained and statistically adequate studies defining the influence of age on cardiac performance during exercise. The existing information has in general been drawn from small subject groups apparently chosen at random without regard to strict age categorization. Standardized work loads have not been employed in testing subjects of various ages, and a variety of techniques such as acetylene, Evans blue dilution, and catheterization for cardiac output estimations have been used. Although considerable work relating to the exercise response, at various ages, of heart rate and blood pressure has been done, very few direct measurements of exercising cardiac output, stroke volume, intra-arterial pressure, and vascular resistance exist (Granath, Jonsson, and Strandell, 1961; Strandell, 1964b). Perhaps even more important, these limited previous studies are almost completely confined to measurements made in subjects supine rather than in their normal upright exercising position. The gross discrepancies between hæmodynamic data obtained supine and upright have now been well defined (Bevegård,

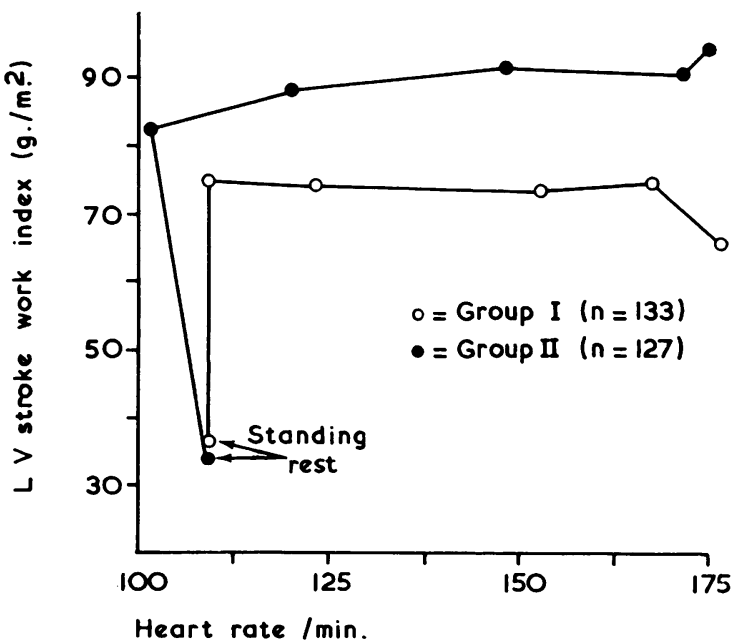

FIG. 6.-Relationship of mean left ventricular stroke work index to heart rate at rest and with increasing work loads. 
Holmgren, and Jonsson, 1960; McGregor, Adam, and Sekelj, 1961; Reeves et al., 1961; Wang, Marshall and Shepherd, 1960), as well as other artefactual variations associated with supine exercise (Frick and Somer, 1964).

It is quite evident from the present study that the response of heart rate, cardiac output, and stroke volume to upright exercise is not compromised or significantly altered in a group of men aged 30-39 years, as compared to a similar group one decade younger. However, the trend observed in the older group towards a lower cardiac output for any given oxygen utilization value may represent a true difference which we might expect to see magnified in the older age-groups now under study. Previous data drawn largely from resting studies (Brandfonbrener, Landowne, and Shock, 1955; Cournand et al., 1945; Landowne, Brandfonbrener, and Shock, 1955; Lewis, 1938; Nickerson, Warren, and Brannon, 1947; Stead et al., 1945) indicate a gradual decline in cardiac output with age, and sparse information on exercise (Granath et al., 1961) tends to confirm this. However, when the exercise-induced increase in cardiac output is related to oxygen consumption, it has been suggested that a comparable response is seen in subjects of widely varying ages (Shock, 1964). The present results, though spanning only a 20 -year age difference, do not tend to support this theory as attested to by divergence of the group linear regression lines for cardiac output plotted against oxygen utilization (Table III). If cardiac output in older age-groups continues to fall, as suggested by existing data, concomitant with an age-associated absolute increase in oxygen utilization for a given work load, this divergence will be magnified. Evidence for this increment in oxygen utilization with age has been suggested by the present material and also in earlier ventilatory studies of 200 men and women equally divided into decades between 20 and 60 years of age (Hanson and Tabakin, 1960, 1961). Significant age increases in this variable have not been delineated in other series (Blackmon et al., 1964; Norris, Shock, and Yiengst, 1955; Norris and Shock, 1957; Strandell, 1964c).

Since the age range of this subject material encompasses 2 groups theoretically "in the prime" of their physically active lives, it is not surprising that age-dependent differences in exercise heart rate response are lacking. Although it is well known that men 50-70 years old will evidence higher heart rates at a given submaximal work load than younger men (Norris, Shock, and Yiengst, 1953; Shock, 1964), that pulse restitution to resting levels will take longer in the older men (Norris et al., 1953), and that maximal heart rates decrease with advancing 20 age (Blackmon et al., 1964; Strandell, 1964a, b), significant heart rate dissimilarity was neither anticipated nor found in this study. Because of this close group correspondence of cardiac output and heart rate, stroke volume of course also varies minimally. Indeed, following initiation of exercise it is seen (Fig. 2) that for all practical purposes both groups reach a stroke volume plateau from which only minor deviations of a few millilitres are observed over the entire exercise range studied. It should perhaps be emphasized again that the relative contributions of heart rate and stroke volume to the increased cardiac output of exercise are quite variable for different subjects. As noted previously (Tabakin et al., 1964), 3 basic types of stroke volume response have been observed in these studies of relatively young men: uniformity over the entire exercise range, level-to-level variations, and a steadily increasing stroke volume with increasing exercise severity. An equal distribution of subjects according to these types of response occurred in both Groups I and II and contributed to the relatively minor variations seen in group means. The evidence strongly supports the thesis that augmentation of stroke volume is not a constant or necessary factor in the cardiac output adaptation to physical stress, at least between the ages of 20 and 40 years.

It has long been recognized that ventilatory volume is larger in older subjects during work, and this is substantiated by the present data, a maximum difference of $81 . / \mathrm{min}$. being observed for Group II at $12^{\circ}$. In comparison to oxygen utilization, ventilation is also seen to be relatively increased, resulting in raised oxygen ventilatory equivalents. From this standpoint Group II's higher absolute oxygen utilization discussed earlier appears more consistent, since in relation to total ventilation oxygen uptake is actually lower than that of the younger men. The same may be said of the higher values for carbon dioxide elimination which, though greater for amount of work performed, are lower per unit of ventilation.

There is little doubt that ageing is normally associated with significant rises in resting blood pressure. Increases of systolic pressure are usually greater than those of diastolic, and a resultant widening of pulse pressure is recorded with advancing years. These changes have usually been attributed to a "decreased volume distensibility of the central vessels" (Landowne et al., 1955; Shock, 1964) associated with an increase in peripheral vascular resistance and partial obliteration of vascular beds with only slight decreases in cardiac output.

Evaluation of blood pressure and peripheral 
resistance changes related to age during exercise has largely been based on arm cuff recordings and a few measurements of cardiac output. In general, moderate pressure increases, predominantly systolic, have been demonstrated in older men, but the physical stress to which both young and old subjects have been exposed has varied greatly and comparisons of responses are thus made difficult. To our knowledge it has not been suggested that these trends may be detectable at relatively early ages and that an age differential of only 10 years may be associated with highly significant pressure increments during even moderate exercise.

That this in fact does occur is shown in Fig. 3. It is evident that primarily systolic but also diastolic and mean pressure differences between the groups are magnified with each added work load. Whereas the younger subjects were able to lower diastolic pressure with successive increases in volume output of the heart, Group II could not thus accommodate, resulting in a rise in mean pressure. It is also of interest to note that though pulse pressure for the two groups is almost identical at rest and during the initial walks, it increases considerably at $8^{\circ}$ and above, thus emphasizing the disproportionate increase in systolic pressure of Group II. The intriguing speculation is, therefore, raised whether these higher degrees of exercise have indeed "prematurely" revealed alterations which previously have been measured only at rest and associated with considerably more advanced age.

Because of the central importance of the arterial blood pressure recordings in the present study, extensive instrumentation system checks were performed. The various transducers, connecting tubings, and pressure amplifiers employed were subjected to repeated analysis of frequency response characteristics and mercury column calibration. Particular scrutiny was applied to mean pressure levels due to the use of this factor in calculation of vascular resistance and ventricular work indices.

Statistical analysis of group mean pressures and their standard deviations by various methods including confidence limits and coefficients of variation all indicate a clear and highly significant separation of the two groups at the $8^{\circ}, 12^{\circ}$, and $14^{\circ}$ exercise levels. There can be little if any doubt that we are dealing with two distinct populations with regard to blood pressure response during performance of large work loads.

It appears most significant that at rest and low exercise levels the systolic, diastolic, and mean arterial pressures in the two groups are quite similar, divergences appearing only at high work loads. Although higher pressure levels than those recorded here in the younger group have been reported, other studies have documented magnitudes comparable to ours (Wang et al., 1960). We cannot be certain what part of these dissimilarities may be related to use of upright exercise, walking rather than bicycling, and other variables appearing in various reports. The more important consideration seems to be that the present 50 subjects were studied by a common method and significant blood pressure differences were found where age categorization was applied.

In view of the observed peripheral resistance differences which carry high statistical significance (Fig. 4), the conclusion must be reached that we are dealing with vascular alterations that have already become established in the 4th decade of life. Increases in resistance of above 12-14 per cent at rest and during moderate exercise are comparable with existing estimates (Landowne et al., 1955) of a 1 per cent per year increase. However, the higher work loads produced resistance increments of 32-54 per cent for the 10-year span studied. Although at rest and during level walking little differentiation of men 20-29 years and those 30-39 years is possible by these measurements or those of heart rate, cardiac output, and ventilatory parameters, these increases in peripheral resistance and blood pressure at higher exercise levels clearly earmark the older men.

These findings are of great significance in terms of the increased ventricular myocardial work for Group II (Fig. 5 and 6). As has been pointed out (Shock, 1964), this represents increased pressure work, and in terms of energy consumption is much more expensive than a comparable increase in kinetic work. Data have been presented (Landowne et al., 1955) that the decrease in cardiac output with ageing outweighs the concomitant increase in pressure, the net result being a reduced left ventricular work. Although this may be true at rest over wide age limits, it does not apply for the present conditions at exercise, despite the relatively young ages investigated.

Despite some obvious defects in "cross-sectional" studies of ageing and its effects on cardiovascular performance, the marked deficiencies in our knowledge of this area justify use of this investigative method. When in 1962 it may be said that "although in recent years it has been possible to study the effects of posture on the cardiac output during exercise, the effects of age .... are still almost completely unexplored" (Wade and Bishop, 1962), any basically sound approach to filling this void is of value. Therefore, though inadvertent bias from the standpoint of mortality and morbidity may play a role in subject selection and not give a pure picture of ageing in contrast to longi- 
tudinal observations, we are presenting a systematic study of certain cardiorespiratory responses to physical stress at various ages. Several factors, we believe, are of merit in this undertaking: subjects are studied in their normal, upright posture; work loads are standardized for all age-groups; a wide spectrum of exercise is used; and commonly accepted and well-standardized methods of measurement are employed. Results of evaluations for the first 2 groups provide evidence for relatively early, significant hæmodynamic alterations with advancing age.

\section{SUMMARY}

Treadmill exercise of graded intensity performed by 50 normal men in the age-groups 20-29 and 30-39 years resulted in significantly higher blood pressures and peripheral resistance values for the older men. Group differences in heart rate, cardiac output, stroke volume, and certain ventilatory variables were minimal, but trends towards lower cardiac output and increased ventilation, oxygen utilization, and carbon dioxide elimination were recorded after the age of 30 . In this group, therefore, maintenance of perfusion and the organism's exercise capacity is achieved at the price of significantly raised ventricular work and energy expenditure.

The authors wish to express their appreciation of the extensive technical assistance of Reba J. Beecher, R. N., Leona Amelia, R. N., and Nellie Cairns.

\section{REFERENCES}

Åstrand, P. O. (1956). Human physical fitness with special reference to sex and age. Physiol. Rev., 36, 307.

Bevegård, S., Holmgren, A., and Jonsson, B. (1960). The effect of body position on the circulation at rest and during exercise, with special reference to the influence on the stroke volume. Acta physiol. scand., 49, 279.

Blackmon, J. R., Doan, A. E., Ansusinha, C., and Bruce, R. A. (1964). Effects of age and cardiovascular disease on maximal exercise capacity in man. Clin. Res., 12, 104.

Brandfonbrener, M., Landowne, M., and Shock, N. W. (1955). Changes in cardiac output with age. Circulation, $12,557$.

Cournand, A., Riley, R. L., Breed, E. S., Baldwin, E. deF., and Richards, D. W. (1945). Measurement of cardiac output in man using the technique of catheterization of the right auricle or ventricle. $\mathcal{f}$. clin. Invest., 24, 106.

Frick, M. H., and Somer, T. (1964). Base-line effects on response of stroke volume to leg exercise in the supine position. f. appl. Physiol., 19, 639.

Granath, A., Jonsson, B., and Strandell, T. (1961). Studies on the central circulation at rest and during exercise in the supine and sitting body position in old men. Acta med. scand., 169, 125.

Hanson, J. S., and Tabakin, B. S. (1960). Carbon monoxide diffusing capacity in normal male subjects, age 20-60, during exercise. f. appl. Physiol., 15, 402. $\longrightarrow$ and - (1961). Steady state carbon monoxide diffusing capacity in normal females. F. appl. Physiol., 16, 839. , and - (1964). Simultaneous and rapidly repeated cardiac output determinations by dye-dilution method. F. appl. Physiol., 19, 275.

$\longrightarrow$, and - (1965). Comparison of the circulatory response to upright exercise in 25 "normal" men and 9 distance runners. Brit. Heart f., 27, 211.

Landowne, M., Brandfonbrener, M., and Shock, N. W. (1955). The relation of age to certain measures of performance of the heart and the circulation. Circulation, $12,567$.

Levy, A. M., Tabakin, B. S., and Hanson, J. S. (1961). Cardiac output in normal men during steady-state exercise utilizing dye-dilution technique. Brit. Heart f., 23, 425.

Lewis, W. H., Jr. (1938). Changes with age in the cardiac output in adult men. Amer. F. Physiol., 121, 517.

McGregor, M., Adam, W., and Sekelj, P. (1961). Influence of posture on cardiac output and minute ventilation during exercise. Circulat. Res., 9, 1089.

Nickerson, J. L., Warren, J. V., and Brannon, E. S. (1947). The cardiac output in man; studies with the low frequency, critically-damped ballistocardiograph, and the method of right atrial catherization. $\mathcal{F}$. clin. Invest., 26, 1.

Norris, A. H., and Shock, N. W. (1957). Age changes in ventilatory and metabolic responses to submaximal exercise. In Fourth Congress of International Assoc. of Gerontology, Merano, Italy, 1957, Vol. 2, p. 512.

$\longrightarrow, \frac{}{,}$ and Yiengst, M. J. (1953). Age changes in heart rate and blood pressure responses to tilting and standardized exercise. Circulation, 8, 521.

$\longrightarrow,-$, and - (1955). Age differences in ventilatory and gas exchange responses to graded exercise in males. f. Geront., 10, 145.

Reeves, J. T., Grover, R. F., Blount, S. G., Jr., and Filley, G. F. (1961). Cardiac output response to standing and treadmill walking. F. appl. Physiol., 16, 283.

Shock, N. W. (1964). Aging of the cardiovascular system. In The Heart and Circulation, 2nd National Conference on Cardiovascular Diseases, Vol. I, Part 2, p. 1204. Washington, D.C. (no publisher shown).

Stead, E. A., Jr., Warren, J. V., Merrill, A. J., and Brannon, E. S. (1945). The cardiac output in male subjects as measured by the technique of right atrial catheterization. Normal values with observations on the effect of anxiety and tilting. $\mathcal{f}$. clin. Invest., 24, 326.

Strandell, T. (1964a). Heart rate and work load at maximal working intensity in old men. Acta med. scand., 176, 301. (1964b). Mechanical systole at rest, during and after exercise in supine and sitting position in young and old men. Acta physiol. scand., 61, 279.

- (1964c). Heart rate, arterial lactate concentration and oxygen uptake during exercise in old men compared with young men. Acta physiol. scand., 60, 197.

Tabakin, B. S., Hanson, J. S., and Levy, A. M. (1965). Effects of physical training on the cardiovascular and respiratory response to graded upright exercise in distance runners. Brit. Heart f., 27, 205.

,,- Merriam, T. W., Jr., and Caldwell, E. J. (1964). Hemodynamic response of normal men to graded treadmill exercise. f. appl. Physiol., 19, 457.

Wade, O. L., and Bishop, J. M. (1962). Cardiac Output and Regional Blood Flow, Chap. 3, p. 51. F. A. Davis, Philadelphia.

Wang, Y., Marshall, R. J., and Shepherd, J. T. (1960). The effect of changes in posture and of graded exercise on stroke volume in man. f. clin. Invest., 39, 1051. 